\title{
Évaluation agronomique de variétés de pays de sainfoin (Onobrychis sativa $L$ ) originaires du sud-est de la France
}

\author{
JM Prospéri ${ }^{1 *}$, F Demarquet ${ }^{2}$, M Angevain ${ }^{1}$, P Mansat ${ }^{1}$ \\ 1 INRA, station de génétique et d'amélioration des plantes, domaine de Melgueil, F34130 Mauguio; \\ 2 Lycée agricole de Carmejane, ferme expérimentale, F04510 Le Chaffaut, France
}

(Reçu le 13 avril 1994; accepté le 20 juin 1994)

\begin{abstract}
Résumé - Trente-quatre variétés de pays de sainfoin collectées dans le sud-est de la France et 17 introductions de pays étrangers ou populations en cours de sélection ont été évaluées en 2 sites principaux : Montpellier et Carmejane (Digne). Les analyses de variance révèlent la supériorité du cultivar Fakir pour la quantité de fourrage produite et la production de semences. Par rapport à ce cultivar, les variétés de pays expriment une croissance hivernale plus importante et sont plus précoces pour le démarrage en végétation ou pour la floraison, tout en conservant un bon niveau de résistance au froid. Cette dernière caractéristique est intéressante car elle permet un pâturage d'hiver qui s'associe bien au rythme d'exploitation des luzernes utilisées dans ces régions. II faut noter le mauvais comportement, dans ces essais, des sainfoins à une coupe (type simple) et notamment leur faible pérennité, à l'inverse de ce qui est habituellement observé. L'analyse en composantes principales, effectuée afin d'identifier des classes de comportement homogène, sépare bien les simples et les doubles, les cultivars étrangers et les variétés de pays, ce qui démontre l'originalité de ces dernières. Une classification ascendante hiérarchique a permis de répartir en 6 classes les origines évaluées. Celles-ci sont décrites par leur valeur agronomique moyenne et sont comparées entre elles. Cette partition servira de base à la constitution de «pools géniques» qui permettront de conserver ces ressources génétiques.
\end{abstract}

Onobrychis sativa $L$ = sainfoin / ressources génétiques / variétés de pays / évaluation agronomique / analyses multivariées

Summary - Agronomic evaluation of local varieties of sainfoin (Onobrychis sativa L) collected in south-east France. Thirty-four local varieties of sainfoin collected in south-east France and 17 accessions from other countries or breeder lines were evaluated in the field at 2 main sites: Montpellier and Carmejane (Digne), over a 3-year life cycle. Two other trials with a smaller number of populations were established in cold situations: Gap (800 m) and Queyras (1 $500 \mathrm{~m}$; near Briançon) to assess the level of winter-hardiness. The following agronomic traits were observed in row nurseries with 3 replications: establishment; winter dormancy; rate of growth; vegetative production; flowering dates; harvest index; and seed production under open-field pollination. An analysis of variance of these traits revealed the superiority of cultivar Fakir for vegetative and seed production. The local varieties showed a better winter growth, a quick start in the spring and a earlier flowering time while keeping a good level of frost tolerance. This is a very interesting trait for the farming systems in these regions, because it allows grazing during the winter which is complementary to the alfalfa cultivars used. Sainfoin shows a large variability for aftermath flowering. The differences between the 2 types of growth, ' 1 cut' and ' 2 cuts', are highly significant. The ' 1 cut' local varieties are less productive and show a low persistency. This is the contrary of what is generally observed by farmers. This could be due to the frequency of cutting (3-4 cuts during the growing season) which is the normal rate for the ' 2 cuts' types. In these regions ' 1 cut' local vari-

\footnotetext{
* Correspondance et tirés à part
} 
eties are usually grazed in spring and autumn, with a rest period during the summer. Principal component analysis was carried out to identify clusters of homogeneous behaviour. Firstly, there is a definite separation between the ' 1 cut' and ' 2 cuts' types and between the cultivars and local varieties. This confirms the originality of these local varieties. A hierarchical clustering method on the first 4 components was used to separate the different groups. A partition into 6 clusters was chosen. Each cluster was described by means of the different traits and compared with the other clusters. The genetic resources of sainfoin are very difficult to preserve, because this species is allogamous, self-incompatible, requires insect pollination, and its seeds have a low viability (5-8 years), even in a cold chamber. These clusters were used to build some 'genetic pools' to preserve the local varieties collected.

Onobrychis sativa $L$ = sainfoin / genetic resources / local varieties / agronomic evaluation / multivariate analysis

\section{INTRODUCTION}

Le sainfoin (Onobrychis sativa $L$ ), plante fourragère de bonne valeur alimentaire, a sa place dans la majorité des systèmes fourragers des exploitations d'élevage des zones de demi-montagne du sud de la France, en haute Provence et sur les Causses. Espèce bien adaptée aux espaces marginaux à faible niveau de fertilité des sols, elle est très appréciée par les éleveurs qui sont unanimes à souligner son intérêt : risque de météorisation faible, croissance plus précoce que la luzerne, bonne appétibilité et digestibilité grâce à un bon équilibre entre glucides et protides. Elle présente une installation facile, même dans des conditions difficiles (sols pierreux, peu profonds, défriche) et valorise particulièrement bien les sols calcaires et secs.

On distingue 2 types agricoles principaux qui correspondent d'après Badoux (1964) aux variétés botaniques bifera et communis. Le premier, ssp bifera, ou sainfoin à 2 coupes (sainfoin à grandes graines, sainfoin chaud, sainfoin double, sainfoin géant), a pour origine le Moyen Orient ; il est désigné ainsi car il a la faculté de remonter à floraison après la première coupe. Le second, ssp communis, ou sainfoin à une coupe (sainfoin commun, sainfoin à petites graines), est originaire de l'Europe centrale ; il est appelé ainsi car les repousses après la première coupe printanière sont uniquement végétatives. II existe, en outre, de nombreux types intermédiaires entre ces 2 extrêmes.

La culture du sainfoin a régressé régulièrement jusqu'à nos jours en Europe. En France, de 700000 ha en 1940, elle est passée à 400000 ha en 1960 à seulement 20000 ha en 1986, presque exclusivement situés dans les départements du sud-est. Les ventes de semences sont passées de 6090 quintaux en 1973 à moins de
1300 quintaux en 1986 pour remonter à 1950 quintaux en 1990 (source GNIS). Les semences utilisées sont, pour l'essentiel, produites sur l'exploitation et disponibles sur un marché local de transactions directes. Des organismes semenciers locaux rassemblent, trient et mettent à disposition des agriculteurs des populations locales. Les raisons de ce déclin sont multiples. Comparativement, la luzerne est plus productive, plus pérenne, utilise mieux les sols riches et fait l'objet d'un large courant de sélection dans le monde. Parallèlement à la régression des surfaces cultivées, on a assisté à l'abandon des recherches et de la sélection sur le sainfoin, excepté dans les pays d'Europe de l'Est.

Pourtant, récemment, un nouvel élan de sélection est apparu en France : son principal motif est une demande accrue de semences de la part des éleveurs de Provence et des Causses. Celleci est provoquée par leur volonté de valoriser au maximum leur territoire avec un matériel végétal bien adapté à leurs besoins et aux milieux défavorisés. Comme pour les autres espèces, une action de sélection vise à améliorer l'adaptation au milieu physique et biotique par l'augmentation des tolérances ou résistances, le niveau de production et les rythmes de croissance. Mais, chez cette espèce, il faut surmonter un handicap : le faible coefficient de multiplication grainière, de l'ordre de 10, alors qu'il atteint 100 pour la luzerne. C'est l'obstacle majeur de la prise en compte de cette espèce par l'industrie de la semence qui explique une bonne part de son déclin.

Afin de soutenir ce nouvel effort de sélection, il convenait d'apprécier la diversité et la valeur des variétés de pays* de sainfoin (ou ressources génétiques), encore multipiiées et cultivées dans le sud-est de la France. Pour ce faire, un programme d'inventaire et de prospection a été réalisé. Il a été conduit par le Groupement de

\footnotetext{
* Dans ce texte, une variété de pays est assimilée à un ensemble de génotypes d'une même espèce sur laquelle un agriculteur d'une région donnée pratique, depuis plusieurs générations, une sélection massale plus ou moins dirigée. Ceci n'interdit pas l'achat de semences provenant d'exploitations agricoles alentours.
} 
recherche et de développement sur le patrimoine génétique (GRD-PAGE PACA) en 1985 et 1986, avec un financement de la région ProvenceAlpes-Côte-d'Azur. Plusieurs origines de sainfoin ont été rassemblées et évaluées tant pour des caractères d'adaptation et de production que de reproduction.

La conservation des ressources génétiques de sainfoin pose de nombreux problèmes. Allogame, autoincompatible et à pollinisation entomophile, le sainfoin nécessite des précautions d'isolement lors des phases de multiplication. Par ailleurs, les semences ont une viabilité réduite (entre 5 et 8 ans), même si elles sont conservées en chambre froide.

L'objectif de cette étude est donc d'évaluer ces ressources génétiques afin d'en faciliter l'utilisation en sélection et de constituer un nombre limité de "pools géniques"» qui seront multipliés à la place des lots d'origine.

\section{MATÉRIELS ET MÉTHODES}

\section{La prospection}

La prospection d'écotypes locaux de sainfoin a été effectuée principalement dans les départements des Alpes-de-Haute-Provence, des Hautes-Alpes et du Vaucluse. Elle a été orientée sur la collecte de semences multipliées par des agriculteurs. Pour identifier les détenteurs de ces "variétés locales", 2 méthodes ont été employées : la diffusion d'une note d'information sur le programme de prospection auprès des mairies rurales, organismes professionnels, agriculteurs et éleveurs connus ; et la prise de contacts directs avec les techniciens et responsables de différents organismes agricoles (EDE, coopératives). À la suite de ces démarches, une collecte de semences a été réalisée lors d'une visite chez les agriculteurs identifiés. Les lots n'ont été prélevés que si les agriculteurs pouvaient préciser l'origine et le régime de multiplication des échantillons. Chaque prélèvement était accompagné d'une fiche d'inventaire sur laquelle figuraient les coordonnées de l'exploitant, la description des techniques de production de semences, les ventes ou achats de semences quand l'information était disponible, ainsi que les caractéristiques écologiques et agronomiques de la parcelle de production d'où venait le lot récolté. Chaque échantillon a été divisé en 2 parties, l'une a été conservée par le conservatoire botanique de Porquerolles en tant que ressource génétique, l'autre a été utilisée pour les évaluations.

\section{Le matériel rassemblé}

L'étude a porté sur les 34 variétés de pays prospectées en quantité suffisante pour être installées dans des essais agronomiques et sur 17 introductions de pays étrangers ou populations en cours de sélection (tableau 1). Ces matériels ont été comparés aux 2 cultivars inscrits sur le catalogue français : Fakir, type à 2 coupes, et Lunique, de type intermédiaire.

\section{Les lieux d'expérimentation}

Les essais ont été implantés aux printemps 1986 et 1987, en 2 lieux contrastés quant à leur environnement climatique (tableau II). Le premier est la station de génétique et d'amélioration des plantes de l'INRA Montpellier, située en plaine littorale, dans une zone de climat méditerranéen sub-humide et frais selon la classification d'Emberger. Les sols d'alluvions calcaires ont un $\mathrm{pH}$ moyen de 7,5. Les essais ont été irrigués durant la période estivale par des apports moyens de $30 \mathrm{~mm}$ par semaine entre juin et septembre. Le second site est le lycée agricole de Carmejane situé à $10 \mathrm{~km}$ de Digne dans la vallée de la Bléone : climat méditerranéen humide et très froid suivant la classification d'Emberger, sols limono-argilocalcaires au pH moyen de 8,0. Les essais n'ont été irrigués que l'année d'implantation entre juin et septembre par des apports de $30 \mathrm{~mm}$ tous les $10 \mathrm{j}$. Deux essais complémentaires de comportement de 10 origines ont été installés au lycée agricole de Gap $(800 \mathrm{~m})$ et dans le parc naturel régional du Queyras $(1500 \mathrm{~m})$ afin de tester les niveaux de tolérance au froid.

\section{Dispositif et notations}

Dans chaque lieu, un essai a été semé au printemps, en 1986 et en 1987, selon un dispositif en blocs randomisés à 3 répétitions. La parcelle élémentaire est constituée de 2 lignes de $2 \mathrm{~m}$ semées à $0,3 \mathrm{~m}$ d'intervalle. Une ligne vide sépare les parcelles élémentaires. Les essais sont restés en place 4 années, ce qui autorise une bonne estimation de la pérennité. Les notations, sur une échelle de 1 à 9 , ont porté sur la rapidité et la vigueur d'installation, la croissance, l'estimation visuelle de la production végétative avant les coupes ou volume, l'intensité de la floraison et de la remontaison, la tolérance au froid, la croissance hivernale et la rapidité de démarrage au printemps. Ces notations ont été complétées, sur le site de Carmejane, par la date de début de floraison et par des mesures de hauteur à la floraison, puis 15 j et 35 j après chaque coupe (moyenne de 6 mesures par parcelle élémentaire). Sur le site de Montpellier, des

\footnotetext{
* Un «pool génique» est la génération résultant d'une multiplication panmictique d'un ensemble de variétés de pays, dans ce cas, ou d'origines génétiques diverses, aux caractéristiques proches.
} 
Tableau I. Origine géographique, type déclaré et numéro des populations installées dans les différents sites d'expérimentation.

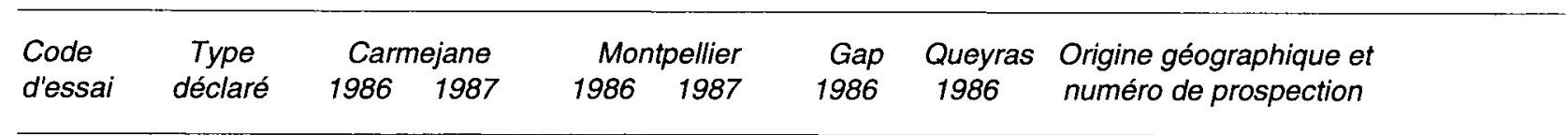

\begin{tabular}{|c|c|c|c|c|c|c|c|c|}
\hline \multicolumn{9}{|c|}{ Dopulations de pays prospectées ou introduites (24 doubles, 10 simples) : } \\
\hline D04D & $\mathrm{D}$ & $x$ & . & $x$ & $\cdot$ & . & $x$ & 04 Monjustin / B02 \\
\hline D04E & $\mathrm{D}$ & $\mathbf{x}$ & . & $\mathrm{x}$ & . & $x$ & $\mathrm{x}$ & 04 La Motte du Caire / B03 \\
\hline D04F & $\mathrm{D}$ & $x$ & . & $\mathrm{x}$ & . & $\mathrm{x}$ & $\mathrm{x}$ & 04 Chateauneuf Val Saint-Donat / B05 \\
\hline D04G & $S$ & $x$ & . & $x$ & . & $x$ & $x$ & 04 Sisteron / B06 \\
\hline $\mathrm{D} 04 \mathrm{H}$ & $\mathrm{D}$ & $x$ & . & $x$ & . & . & . & 04 La Begude 1 / C01 \\
\hline D04I & $\mathrm{D}$ & $x$ & . & $x$ & . & $x$ & $x$ & 04 La Begude 2 / C02 \\
\hline D04J & $\mathrm{D}$ & $x$ & . & $x$ & . & . & . & 04 Saint-Jurs / C04 \\
\hline D04K & $\mathrm{D}$ & $x$ & . & $x$ & . & . & . & 04 Allons / C06 \\
\hline D04L & $\mathrm{D}$ & $x$ & . & $x$ & . & $x$ & $x$ & 04 Sourribes / B10 \\
\hline D04M & $\mathrm{D}$ & $x$ & . & $x$ & . & . & . & 04 Saint-Michel Observatoire / 809 \\
\hline D04N & $D$ & $x$ & . & . & . & . & . & 04 Mezel / BLANC \\
\hline D040 & $S$ & $x$ & . & $x$ & . & $x$ & $x$ & 04 Sisteron / Vaumeilh / B01-C08 \\
\hline D04P & $?$ & $x$ & . & $x$ & . & . & . & 04 La Begude 3 / B13 \\
\hline D04Q & $?$ & . & $x$ & $x$ & $x$ & . & . & 04 Indéterminée / B11 \\
\hline D04R & $?$ & . & $x$ & . & $x$ & . & . & 04 Vallée du Verdon \\
\hline D05A & $\mathrm{D}$ & $x$ & 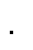 & $x$ & . & . & . & 05 Serres / Le Bersac 1/Ch1 \\
\hline D05B & $\mathrm{D}$ & $x$ & . & $x$ & . & . & . & 05 Serres / Savournon 1/Ch9 \\
\hline D05C & $\mathrm{D}$ & $x$ & . & $x$ & . & . & . & 05 Serres / Le Bersac 2 : Ch2 \\
\hline D11A & $\mathrm{D}$ & $x$ & . & $x$ & . & $x$ & $x$ & 11 Ets Carneau \\
\hline D84A & $\mathrm{D}$ & . & $x$ & . & $x$ & . & . & 84 La Bastide des Jourdans / L01 \\
\hline D84B & $\mathrm{D}$ & . & $x$ & . & $x$ & . & . & 84 Roussillon / L10 \\
\hline D84C & $\mathrm{D}$ & . & $x$ & . & $x$ & 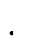 & . & 84 Saignon / Les Bruns / L17 \\
\hline D84D & $\mathrm{D}$ & . & $x$ & . & $x$ & & . & 84 Caseneuve / L18 \\
\hline D84E & $S$ & . & $x$ & . & $x$ & . & . & 84 Aurel / Les Gardes / L07 \\
\hline SO4C & $S$ & $x$ & . & $x$ & . & . & . & 04 Vachères / B04 \\
\hline D04D & $S$ & $x$ & . & $x$ & . & $x$ & $x$ & 04 Puimoisson / C03 \\
\hline SO4E & $S$ & $x$ & . & $x$ & . & $x$ & $x$ & 04 Plateau de Valensole / C05 \\
\hline S04G & $?$ & $x$ & . & $x$ & . & . & . & 04 Ets Michel \\
\hline $\mathrm{SO} 4 \mathrm{H}$ & $?$ & $x$ & . & $x$ & . & . & . & 04 Indéterminée / C07 \\
\hline S05A & $\mathrm{S}$ & $x$ & . & $x$ & . & . & . & 05 Serres / Sigottier 1 / Ch6 \\
\hline S05B & $\mathrm{S}$ & $x$ & . & $x$ & . & . & . & 05 Serres / Sigottier 2 / Ch4 \\
\hline S05C & $\mathrm{S}$ & $x$ & . & $x$ & . & . & . & 05 Serres / Savournon 2 / Ch5 \\
\hline S84C & $S$ & . & $x$ & . & $x$ & . & . & 84 Viens / le Canet / L08 \\
\hline S84D & $\mathrm{S}$ & . & $x$ & . & $x$ & . & . & 84 Viens / Les Sybilles / L14 \\
\hline
\end{tabular}

Matériels sélectionnés et cultivars témoins (11 doubles, 4 intermédiaires, 2 simples) :

\begin{tabular}{|c|c|c|c|c|c|c|c|c|}
\hline \\
\hline D06A & $\mathrm{D}$ & $\mathrm{x}$ & . & $x$ & & . & . & Sélection Antibes 1 \\
\hline D06B & $\mathrm{D}$ & $x$ & . & $x$ & & . & . & Sélection Antibes 2 \\
\hline D06C & D & $x$ & . & $\mathrm{x}$ & & . & . & Sélection Antibes 3 \\
\hline DEsA & $?$ & . & $x$ & . & $x$ & . & . & Espagne / Zaragoza \\
\hline $\mathrm{DHOA}$ & $?$ & . & $x$ & . & $x$ & . & . & Hongrie / Komploti \\
\hline DHoB & ? & . & $\mathrm{x}$ & . & & . & . & Hongrie / Matra \\
\hline DPoA & $?$ & $\mathrm{x}$ & . & $\mathrm{x}$ & . & . & . & Pologne / Perska \\
\hline DTCA & $?$ & $x$ & . & $\mathrm{x}$ & . & . & . & Tchécoslovaquie / Buciansky 1 \\
\hline DTcB & $?$ & $x$ & . & $\mathrm{x}$ & & 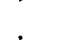 & . & Tchécoslovaquie / Buciansky 2 \\
\hline DTcC & $?$ & $x$ & . & $x$ & . & 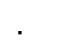 & . & Tchécoslovaquie / Visnovsky \\
\hline FAKI & $\mathrm{D}$ & $x$ & $x$ & $\mathrm{x}$ & $x$ & $\mathrm{x}$ & $\mathrm{x}$ & Fakir (INRA Montpellier) \\
\hline IGrA & $?$ & $x$ & . & . & . & 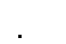 & . & Grèce / Sélection SK48 \\
\hline ITUA & $?$ & $x$ & . & $x$ & . & . & . & Turquie / indéterminée \\
\hline ITUB & $?$ & $x$ & - & . & . & . & . & Turquie / Esenbaja \\
\hline LUNI & 1 & $x$ & . & $x$ & . & . & . & Lunique (INRA Lusignan) \\
\hline S06A & $\mathrm{s}$ & $x$ & . & $x$ & . & . & . & Sélection Antibes 4 \\
\hline SPoB & $?$ & . & $x$ & $x$ & . & . & . & Pologne / Shsessowicka \\
\hline otal & & 38 & 14 & 37 & 12 & 10 & 11 & \\
\hline
\end{tabular}


Tableau II. Caractéristiques climatiques moyennes sur un minimum de 25 années des stations météorologiques les plus proches des sites d'expérimentation.

Station météo Altitude $(m)$ Précipitation annuelle Pluies d'hiver (nov-avril) Moyenne minima de janvier

\begin{tabular}{lrrrr}
\hline Montpellier (34) & 3 & 690 & 360 & $1,8^{\circ} \mathrm{C}$ \\
Carmejane (04) & 550 & 710 & 340 & $-4,0^{\circ} \mathrm{C}$ \\
Gap (05) & 780 & 930 & 470 & $-4,8^{\circ} \mathrm{C}$ \\
Briançon (05) & 1450 & 760 & 325 & ND \\
\hline
\end{tabular}

Source : Météorologie nationale. ND : données non disponibles.

mesures des composantes du rendement grainier ont été faites sur 10 tiges, sur chaque parcelle élémentaire en 1987 et 1988, c'est-à-dire en deuxième et troisième année.

\section{Les méthodes d'analyses}

Des analyses de variance ont été réalisées séparément sur les données de chaque site pour connaître la signification des effets bloc, variété ou type de croissance (simple ou double). Les données n'ont pu être analysées globablement sur les 2 sites en raison de la forte hétérogénéité dans les cycles de développement résultant d'environnements climatiques trop éloignés (date de démarrage au printemps, irrigation estivale, ...) et du nombre différent de variétés testées. Pour synthétiser les effets variétés et sites d'expérimentation, une analyse en composantes principales a été réalisée sur les variables mesurées sur les essais menés en 1986 à Montpellier et Carmejane. La valeur moyenne des 3 répétitions a été affectée à chaque variété de pays. Les notations retenues pour les sites de Carmejane et de Montpellier constituent des variables différentes. Cette analyse porte sur les 35 variétés communes aux 2 essais.

\section{RÉSULTATS ET DISCUSSION}

\section{Les analyses de variances}

\section{Effet variété}

La moyenne, le minimum et le maximum des principales variables mesurées sont données sur le tableau III pour les essais de Montpellier et sur le tableau IV pour les essais de Carmejane. La majorité des variables présente un effet variété très significatif, à l'exception du nombre d'inflorescences par tige qui est extrêmement variable à l'intérieur d'une origine, d'une plante à l'autre ou d'une tige à l'autre. Ceci est corroboré par les observations de Carleton et Wiesner (1968) qui ont trouvé des productions de semences variant de 1 à 23 entre différentes plantes clonées issues de la variété commerciale de sainfoin Eski.

Ces variétés, maintenues par sélection massale par les agriculteurs de cette région, montrent des performances agronomiques inférieures, ou au mieux légèrement supérieures à celles du témoin Fakir, notamment pour les variables de production végétative. Cela apparaît aussi bien sur les essais de Montpellier que de Carmejane. Sur l'essai de Montpellier, où le climat hivernal clément permet l'expression d'une croissance hivernale importante, ces origines du sud-est sont en moyenne plus poussantes en fin d'automne et plus précoces pour le démarrage de végétation au printemps que le témoin Fakir, bien que non significativement différentes de celle-ci, à l'exception de la croissance hivernale mesurée en décembre 1986. Cette bonne croissance hivernale est associée à une floraison plus précoce sur le premier cycle. Sur l'essai de Carmejane semé en 1986, 27 variétés sur les 38 testées sont significativement plus précoces que le témoin Fakir au printemps 1987. La plus précoce fleurit $10 \mathrm{j}$ avant Fakir. Ces différences s'atténuent nettement à partir de la $3^{e}$ année, car la baisse de densité, due à la faible pérennité des plantes de sainfoin, augmente fortement la variation intra-variété et les seuils de signification de l'essai. Aucune notation n'a pu être réalisée sur ces essais à partir de 1990 en raison de leur trop forte hétérogénéité.

Negri et al, en 1987, avaient trouvé une forte variation du poids de 1000 gousses sur des écotypes naturels de sainfoin, entre 9 et $18 \mathrm{~g}$. Dans notre expérimentation, les différences entre les variétés de pays ne sont pas significatives et leur poids de 1000 gousses est élevé, entre 20 et 28 
Tableau III. Effet de la population et statistiques élémentaires pour les principales notations réalisées sur les essais de Montpellier.

$\begin{array}{ccccc}\text { Moyenne Min-Max } & \begin{array}{c}\text { Effet } \\ \text { variété }\end{array} & \begin{array}{l}\text { Effet } \\ \text { bloc }\end{array} & \text { Fakir } & \begin{array}{c}\text { Nombre de variétés } \\ \text { >Fakir }\end{array}\end{array}$

\section{Montpellier semis 1986}

\begin{tabular}{|c|c|c|c|c|c|c|c|}
\hline Densité de levée & 6,88 & $2,0-8,5$ & ** & ** & 8,3 & & $4(3 S)$ \\
\hline Volume $1^{\text {re }}$ coupe 86 & 5,43 & $3,0-7,5$ & $\star \star$ & NS & 6,8 & & $6(4 S)$ \\
\hline Volume $2^{e}$ coupe 86 & 5,28 & $2,5-7,5$ & NS & $\star \star$ & 5,5 & & \\
\hline Volume $2^{e}$ coupe 87 & 4,99 & $2,0-9,0$ & $\star \star$ & NS & 6,0 & 4 & 12 (9S) \\
\hline Volume $3^{e}$ coupe 87 & 6,31 & $4,5-8,5$ & ** & NS & 6,5 & & \\
\hline Volume $1^{\text {re }}$ coupe 88 & 6,37 & $3,5-9,0$ & $\star \star$ & $\star \star$ & 8,3 & & $13(10 S)$ \\
\hline Volume $1^{\text {re }}$ coupe 89 & 5,67 & $2,0-9,0$ & $\star *$ & $\star \star$ & 6,3 & 4 & $10(8 S)$ \\
\hline $\mathrm{CH}$ décembre 86 & 7,08 & $3,0-9,0$ & 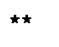 & NS & 6,3 & $17(5 S)$ & 2 \\
\hline $\mathrm{CH}$ février 87 & 6,69 & $3,0-9,0$ & ** & ** & 6,3 & 5 & 2 \\
\hline CH décembre 88 & 5,77 & $2,0-9,0$ & $\star \star$ & NS & 7,0 & & 13 (9S) \\
\hline $\mathrm{CH}$ février 89 & 5,44 & $2,0-9,0$ & $\star \star \star$ & NS & 5,5 & 7 & $6(4 S)$ \\
\hline Remontaison juillet 1987 & 3,39 & $1,0-8,0$ & ** & NS & 2,5 & 14 & \\
\hline Remontaison septembre 1987 & 1,64 & $1,0-2,0$ & $\star \star$ & NS & 2,0 & & $12(10 S)$ \\
\hline Nombre Inflo 87 par tige & 3,10 & $2,4-3,7$ & NS & NS & 3,3 & & \\
\hline Nombre Inflo 88 par tige & 3,85 & $3,0-5,2$ & NS & NS & 4,3 & & \\
\hline Longueur moyenne Inflo 87 & 7,88 & $5,3-10,6$ & $\star \star$ & * & 7,9 & 1 & 1 \\
\hline Longueur moyenne Inflo 88 & 7,66 & $6,0-9,6$ & $\star \star$ & NS & 8,9 & & $5(2 S)$ \\
\hline Nombre gousses 87 par Inflo & 11,9 & $7,0-22,0$ & $\star \star$ & $\star \star$ & 11,3 & 2 & \\
\hline Nombre gousses 88 par Inflo & 13,7 & $6,4-23,0$ & $\star \star$ & $\star \star$ & 14,5 & 2 & $2(2 S)$ \\
\hline Poids 1000 Gousses 87 & 23,8 & $20,2-28,6$ & NS & NS & 24,7 & & \\
\hline Densité février 89 & 6,18 & $2,5-9,0$ & $\star \star$ & NS & 6,5 & & $3(2 S)$ \\
\hline
\end{tabular}

Montpellier semis 1987

Intensité floraison 88
Volume 1 re coupe 88
$\mathrm{CH}$ décembre 88
$\mathrm{CH}$ février 89
Densité février 89

$\begin{array}{ll}4,65 & 0,5-9,0 \\ 6,46 & 3,5-8,5 \\ 5,84 & 4,0-8,0 \\ 5,11 & 3,5-7,0 \\ 6,08 & 5,5-7,0\end{array}$

$\begin{array}{lll}\text { NS } & \text { NS } & 2,0 \\ \star & * \star & 8,5 \\ \text { NS } & \star \star & 5,5 \\ \star & \text { NS } & 4,5 \\ \text { NS } & * & 6,0\end{array}$

En 1986, 37 populations testées : 10 simples, 2 intermédiaires, 25 doubles ; en 1987, 12 populations testées : 2 simples, 10 doubles. $\mathrm{CH}=$ croissance hivernale, Inflo = inflorescence. Les notations de volume (estimation de la production végétative), de croissance hivernale et de densité ont été faites sur une échelle de 1 à 9 . Les mesures sur les inflorescences ont été réalisées sur 10 tiges par parcelle élémentaire. L'importance des remontaisons est notée de 1 à 5 . Min : minimum, Max : maximum. Nombre de populations significativement supérieures ou inférieures au témoin Fakir au seuil de $5 \%$ (test de Duncan), entre parenthèses le nombre de populations de type simple. NS : non significatif, ${ }^{*}$ : significatif au seuil de $5 \%,{ }^{* *}$ : significatif au seuil de $1 \%$.

g. Il existe donc une sélection involontaire (mécanisation) ou volontaire de la part des agriculteurs pour maintenir une production de semences lourdes. La production grainière de ces variétés de pays est très faible et inférieure à celle de Fakir; seules les introductions DPoA (Pologne) et DTcC (Tchécoslovaquie) ont une production de semences équivalente à celle de Fakir.

La résistance au froid de ces populations de pays de sainfoin précoces est tout à fait satisfaisante puisque aucun dégât de destruction de plantes n'a été constaté, et ce, malgré la rigueur particulière de I'hiver 1986-1987. Les origines installées dans les situations les plus extrêmes, à Gap et dans le Queyras, n'ont subi aucun dégât de froid durant l'hiver et n'ont manifesté qu'une faible sensibilité des organes reproducteurs lors d'un gel tardif en juin 1987.

\section{Effet type de croissance}

Le sainfoin présente une variation très nette entre origines pour la capacité à remonter à floraison. Au sein d'une même population, il peut 
Tableau IV. Effet de la population et statistiques élémentaires pour les principales notations réalisées sur les essais de Carmejane.

$\begin{array}{ccccc}\text { Moyenne Min-Max } & \text { Effet } & \text { Effet } \\ \text { variété } & \text { bloc } & \text { Fakir } \begin{array}{c}\text { Nombre de variétés } \\ \text { >Fakir < <akir }\end{array}\end{array}$

\section{Carmejane semis 1986}

\begin{tabular}{|c|c|c|c|c|c|c|c|}
\hline Densité 1 mois après levée & 50,1 & $9,0-71,0$ & $\star \star$ & $\star \star \star$ & 55,3 & & $4(1 S)$ \\
\hline Hauteur 1 mois après levée & 8,7 & $4,9-12,2$ & ** & ** & 8,8 & & \\
\hline Croissance mars 87 & 5,27 & $1,0-7,3$ & ** & $\star \star \star$ & 3,33 & $6(2 S)$ & \\
\hline Croissance avril 87 & 5,47 & $1,7-8,3$ & $\star \star$ & ** & 4,00 & 4 & \\
\hline Hauteur avril 88 & 34,5 & $25,9-40,6$ & $* \star$ & NS & 33,9 & 3 & 1 \\
\hline Hauteur floraison 87 & 79,6 & $56,5-97,0$ & $\star \star$ & NS & 92,1 & & $17(9 S)$ \\
\hline Hauteur floraison 88 & 87,7 & $69,7-115,1$ & $\star *$ & NS & 88,0 & $1(1 S)$ & \\
\hline Hauteur floraison 89 & 66,0 & $46,8-81,4$ & $\star \star$ & NS & 72,0 & & $10(8 S)$ \\
\hline Hauteur $2^{\mathrm{e}}$ coupe 86 & 42,9 & $19,1-64,4$ & $\star \star$ & ** & 62,4 & & $23(9 S)$ \\
\hline Hauteur $2^{\mathrm{e}}$ coupe 87 & 56,6 & $30,6-73,6$ & $\star \star$ & NS & 71,8 & & $11(9 S)$ \\
\hline Hauteur $2^{e}$ coupe 88 & 43,7 & $24,0-59,8$ & $\star \star *$ & $\star \star \star$ & 52,8 & 1 & $17(9 S)$ \\
\hline Hauteur $2^{e}$ coupe 89 & 44,0 & $24,0-57,6$ & ** & ** & 53,6 & & $15(9 S)$ \\
\hline Hauteur $3^{e}$ coupe 86 & 23,3 & $14,7-28,9$ & ** & ** & 28,2 & & $24(8 S)$ \\
\hline Hauteur $3^{e}$ coupe 87 & 42,7 & $26,3-63,7$ & ** & $\star \star$ & 59,3 & & $28(9 S)$ \\
\hline Hauteur $3^{e}$ coupe 88 & 19,4 & $11,0-27,4$ & $\star *$ & $\star \star$ & 22,8 & 2 & 17 (9S) \\
\hline Hauteur $4^{e}$ coupe 87 & 21,6 & $13,7-30,0$ & $\star \star$ & $\star \star *$ & 26,7 & 1 & $32(9 S)$ \\
\hline Date de floraison 87 & 24,3 & $19,0-32,3$ & $\star *$ & * & 29,3 & 2 & 27 (9S) \\
\hline Date de floraison 88 & 20,3 & $11,7-28,3$ & $\star \star$ & NS & 23,3 & 3 & $11(9 S)$ \\
\hline Date de floraison 89 & 12,6 & $5,8-24,0$ & $\star \star$ & $\star \star$ & 13,0 & 7 & $9(9 S)$ \\
\hline Remontaison août 86 & 2,57 & $1,0-4,7$ & ** & $\star \star \star$ & 3,33 & 6 & 14 (9S) \\
\hline Remontaison juillet 87 & 2,19 & $1,0-4,0$ & $\star \star$ & NS & 2,00 & 6 & \\
\hline Remontaison septembre 87 & 1,54 & $1,0-2,0$ & ** & * & 2,00 & & $16(9 S)$ \\
\hline Remontaison juillet 88 & 3,67 & $1,0-5,0$ & 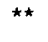 & NS & 5,00 & & $12(9 S)$ \\
\hline Remontaison août 88 & 2,57 & $1,0-5,0$ & ** & * & 4,00 & & $13(9 S)$ \\
\hline $\mathrm{Nb}$ Inflo $87 / \mathrm{ml}$ & 89,6 & $51,7-115,3$ & ** & * & 84,7 & & \\
\hline Densité juillet 89 & 2,9 & $1-4$ & ** & * & 3 & & $3(1 S)$ \\
\hline \multicolumn{8}{|l|}{ Carmejane semis 1987} \\
\hline Densité 1 mois après levée & 44,1 & $25,0-62,0$ & ** & NS & 45,0 & & \\
\hline Hauteur 1 mois après levée & 9,7 & $5,8-11,8$ & ** & NS & 10,1 & 1 & $1(1 S)$ \\
\hline Hauteur floraison 88 & 102,5 & $82,9-118,3$ & $\star \star *$ & NS & 103,4 & 3 & $3(35)$ \\
\hline Hauteur floraison 89 & 72,1 & $44,2-83,2$ & $\star \star$ & NS & 73,0 & & $3(3 S)$ \\
\hline Hauteur floraison 90 & 67,5 & $46,4-82,6$ & $\star \star \star$ & NS & 70,2 & & $6(3 S)$ \\
\hline Hauteur $2^{\mathrm{e}}$ coupe 87 & 26,5 & $15,2-36,3$ & $\star \star \star$ & NS & 36,3 & & $13(3 S)$ \\
\hline Hauteur $2^{\mathrm{e}}$ coupe 88 & 47,7 & $26,2-63,3$ & $* *$ & * & 63,3 & & 12 (3S) \\
\hline Hauteur $2^{\mathrm{e}}$ coupe 89 & 50,9 & $28,5-64,6$ & $\star \star \star$ & * & 64,6 & & $10(3 S)$ \\
\hline Hauteur $2^{\circ}$ coupe 90 & 30,7 & $13,6-42,2$ & $\star \star \star$ & * & 39,7 & & $10(3 S)$ \\
\hline Hauteur $3^{e}$ coupe 88 & 23,8 & $14.2-28.8$ & ** & NS & 26,9 & & $7(3 S)$ \\
\hline Date de floraison 88 & 19,9 & $10,0-28,3$ &  & NS & 27,3 & & $10(3 S)$ \\
\hline Date de floraison 89 & 13,0 & $4,3-22,7$ & $\star \star$ & $\star \star$ & 18,7 & 2 & $11(3 S)$ \\
\hline Densité juillet 89 & 2,5 & $1,3-3,0$ & * & NS & 2,7 & & 1 \\
\hline
\end{tabular}

En 1986, 38 populations testées : 9 simples, 4 intermédiaires, 25 doubles ; en 1987, 14 populations testées : 3 simples, 11 doubles. La densité un mois après la levée est exprimée en nombre de plantes par mètre linéaire. Les hauteurs sont mesurées en centimètres après $30 \mathrm{j}$ de repousse (moyenne de 6 mesures par parcelle élémentaire). Les dates de floraison sont exprimées en nombre de jours après le 1er mai. $\mathrm{Nb}$ Inflo $87 / \mathrm{ml}$ : nombre d'inflorescences par mètre linéaire en 1987. La densité de juillet 1989 est notée de 1 à 4 . L'importance des remontaisons est notée de 1 à 5 . Min = minimum, Max = maximum. Nombre de populations significativement supérieures ou inférieures au témoin Fakir au seuil de $5 \%$, entre parenthèses le nombre de populations de type simple. NS : non significatif, " : significatif au seuil de $5 \%$, ** : significatif au seuil de $1 \%$. 
exister des différences importantes entre plantes (Marie, 1961). Il existe une continuité entre les 2 types extrêmes parmi les variétés de pays. Une vérification du type de croissance déclaré par les agriculteurs lors de la prospection a pu être faite à partir des notations de remontaison après la première coupe, réalisées en 1987, 1988 et 1989. Ces notations sont très cohérentes entre les sites d'expérimentation. II a été possible de classer les origines en 3 groupes : un type simple (S) présentant aucune ou très peu de remontaison, avec moins de $5 \%$ de plantes remontantes ; un type intermédiaire (I) avec une remontaison faible dont 5 à $50 \%$ des plantes peuvent fleurir lors du $2^{e}$ cycle ; un type double (D) présentant une forte remontaison dont plus de $50 \%$ de plantes fleurissent lors du $2^{\mathrm{e}}$ cycle. La première lettre du code d'essai mentionné sur le tableau I indique à quel type de croissance est rattaché la variété de pays. Celui-ci est en général conforme aux déclarations faites par l'agriculteur lors de la collecte, sauf pour les origines D040 et D84E, déclarées en type simple et qui sont en fait des types doubles. Cinq lots issus de la prospection et 11 issus d'introduction, dont le type de croissance était inconnu, ont pu être aisément classés.

L'effet du type de croissance est très net sur toutes les variables mesurées (tableau $\mathrm{V}$ ). Les sainfoins de type simple sont significativement moins productifs et moins poussants que les doubles ; la différence s'accentue à partir de la $2^{\mathrm{e}}$ coupe. Chez les types simples, la forte diminution de croissance des tiges lors des $2^{e}$ et $3^{e}$ cycles, due au faible pourcentage de remontaison, entraîne un déficit global de production de matière sèche par rapport aux types doubles.

Il est traditionnellement admis que les sainfoins de type double sont moins pérennes que ceux de type simple (Lapeyronnie, 1982 ; Broucqsault et Feraud, 1990 ; GNIS, 1991). Cooper, en 1972, ne note aucune différence entre des variétés de type simple et double. Ici, il est curieux de constater que, dans tous les essais installés, les origines de type simple sont nettement moins pérennes que celles de type double. Une partie de l'explication est fournie par la faible qualité des semences des types simples. On note, en effet, sur les essais une plus faible levée de ces origines et un déficit de production dès la première année. Une autre partie de l'explication peut être l'introgression de caractéristiques de Fakir, variété sélectionnée de type double et de bonne pérennité, dans ces variétés de pays. Cette variété a été très largement commercialisée, diffusée et multipliée dans ces régions agricoles françaises. L'introgression est très difficile à déceler chez les types doubles. Chez les sainfoins simples, toute introgression de la variété Fakir, même légère, s'accompagne d'une modification du type de croissance et entraîne automatiquement un classement de ces variétés en type double ou en type intermédiaire. Néanmoins le niveau d'introgression reste faible car les variétés de pays de type double se distinguent assez nettement de Fakir pour leur précocité. Une troisième hypothèse, plus vraisemblable, réside dans le fait que le rythme d'exploitation en fauche favoriserait les types doubles. En effet, dans les systèmes culturaux de ces régions, les types simples sont traditionnellement pâturés au début du printemps et bénéficient d'un repos estival marqué.

\section{L'analyse en composantes principales}

L'analyse en composantes principales (ACP) est maintenant largement utilisée pour synthétiser des résultats quels que soient les dispositifs retenus : en plantes isolées (Negri et al, 1987 ; Charmet et Balfourier, 1991), en parcelles denses sur un ou plusieurs lieux (Charmet et al, 1989 ; Volaire et al, 1992).

Le tableau VI exprime les coefficients de corrélation entre les 46 variables retenues (21 pour le site de Montpellier et 25 pour le site de Carmejane) et les 4 premières composantes principales. Le premier plan explique $65 \%$ de la variance totale, les 4 premiers axes, plus de $76 \%$. Le premier axe peut être interprété comme un axe de vigueur puisque toutes les notations d'estimation de la production et de la hauteur lui sont très fortement négativement corrélées. Les notations de remontaison sont, elles aussi, fortement liées à cet axe. Ceci confirme la relation que l'on avait notée entre le type de croissance et la vigueur lors des analyses de variance. On observe, sur l'axe 2, une opposition entre les dates de floraison, mesurées à Carmejane, corrélées positivement à cet axe et les observations de croissance hivernale, mesurées en 1987 sur les 2 sites, qui lui sont corrélées négativement. Cet axe peut donc être interprété comme un axe de précocité de floraison et de démarrage au printemps. L'axe 1 discrimine bien les types simples et doubles. L'axe 2 sépare bien tous les cultivars sélectionnés (Fakir, Lunique ou les différentes introductions) des variétés de pays (fig 1). 
Tableau V. Effet du type de croissance pour les principales notations réalisées sur les essais de Montpellier 1986 et de Carmejane 1986.

Moyenne Effet type Simple Double

\section{Montpellier semis 1986}

$\begin{array}{lr}\text { Densité de levée } & 7,04 \\ \text { Volume } 1^{\text {re coupe } 86} & 5,52 \\ \text { Volume } 2^{e} \text { coupe } 86 & 5,39 \\ \text { Volume } 2^{\mathrm{e}} \text { coupe } 87 & 5,11 \\ \text { Volume } 3^{e} \text { coupe } 87 & 6,46 \\ \text { Volume } 1^{\text {re }} \text { coupe } 88 & 6,42 \\ \text { Volume 1re coupe } 89 & 5,94 \\ \mathrm{CH} \text { décembre } 86 & 7,44 \\ \mathrm{CH} \text { février } 87 & 7,00 \\ \mathrm{CH} \text { décembre } 88 & 6,04 \\ \text { CH février } 89 & 5,74 \\ \text { Remontaison juillet } 87 & 3,54 \\ \text { Remontaison septembre } 87 & 1,69 \\ \text { Nombre Inflo } 87 \text { par tige } & 3,06 \\ \text { Nombre Inflo } 88 \text { par tige } & 3,81 \\ \text { Longueur moyenne Inflo } 87 & 7,67 \\ \text { Longueur moyenne Inflo } 88 & 7,48 \\ \text { Nombre gousses } 87 \text { par Inflo } & 11,6 \\ \text { Nombre gousses } 88 \text { par Inflo } & 13,8 \\ \text { Poids 1 ooo Gousses } 87 & 23,8 \\ \text { Densité février } 89 & 6,37\end{array}$



$\begin{array}{lcc}\star \star & 6,19 & 7,43 \\ \text { NS } & 4,69 & 5,91 \\ \star \star & 5,46 & 5,36 \\ \text { NS } & 2,46 & 6,34 \\ \text { NS } & 5,46 & 6,93 \\ \star \star & 4,46 & 7,35 \\ \star & 3,92 & 6,88 \\ \text { NS } & 7,19 & 7,55 \\ \text { * } & 6,54 & 7,21 \\ \text { NS } & 3,96 & 7,00 \\ \text { NS } & 3,92 & 6,59 \\ \text { NS } & 1,00 & 4,71 \\ \text { NS } & 1,04 & 2,00 \\ \text { NS } & 3,00 & 3,09 \\ \text { NS } & 4,08 & 3,68 \\ \text { NS } & 7,86 & 7,58 \\ \text { NS } & 7,30 & 7,56 \\ \star \star & 9,0 & 12,8 \\ \star \star & 9,2 & 16,0 \\ \text { NS } & 23,3 & 24,1 \\ \text { NS } & 4,58 & 7,20\end{array}$

\section{Carmejane semis 1986}

Densité 1 mois après levée

Hauteur 1 mois après levée

Croissance mars 87

Croissance avril 87

Hauteur avril 88

Hauteur floraison 87

Hauteur floraison 88

Hauteur floraison 89

Hauteur $2^{\mathrm{e}}$ coupe 86

Hauteur $2^{\theta}$ coupe 87

Hauteur $2^{\mathrm{e}}$ coupe 88

Hauteur $2^{e}$ coupe 89

Hauteur $3^{8}$ coupe 86

Hauteur $3^{\circ}$ coupe 87

Hauteur $3^{\circ}$ coupe 88

Hauteur $4^{e}$ coupe 87

Date de floraison 87

Date de floraison 88

Date de floraison 89

Remontaison août 86

Remontaison juillet 87

Remontaison septembre 87

Remontaison juillet 88

Remontaison août 88

$\mathrm{Nb}$ Inflo $87 / \mathrm{ml}$

Densité juillet 89
53,2

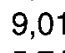

5,71

5,82

35,1

78,6

87,1

66,3

44,2

56,4

44,5

43,8

24,1

43,9

19,8

22,4

23,9

19,7

12,2

2,68

2,28

1,60

3,73

2,76

88,7

3,06
48,7

7,38

5,67

5,13

31,3

61,7

75,5

53,7

25,1

34,5

28,0

28,7

23,0

27,5

15,9

20,6

19,2

13,1

7,7

1,00

1,15

1,00

1,13

1,00

100,7

2,67
55,3

9,81

5,73

6,15

37,0

86,8

92,7

72,5

53,4

66,9

52,5

50,6

24,7

51,7

21,5

23,3

26,1

22,8

14,4

3,48

2,83

1,89

4,99

3,61

83,0

3,25

Pour les variables mesurées, voir tableaux III et IV. 
Tableau VI. Coefficients de corrélation entre les variables initiales, mesurées à Montpellier et Carmejane pour les essais semés en 1986, et les 4 premiers facteurs de l'analyse en composantes principales.

Axe 1

Axe 2

Axe 3

Axe 4

Montpellier semis 1986

Densité 1 mois après levée

Volume 1re coupe (26/06/96)

Volume $2^{e}$ coupe $(18 / 07 / 86)$

Volume $2^{e}$ coupe $(20 / 07 / 87)$

Volume $3^{e}$ coupe (04/09/87)

Volume tre coupe (28/04/88)

Volume tre coupe (19/03/89)

Croissance au 15/12/86

Croissance au 17/02/87

Croissance au 05/12/88

Croissance au 16/02/89

Remontaison juillet 1987

Remontaison septembre 1987

Nombre inflorescences / tige 1987

Nombre inflorescences / tige 1988

Longueur inflorescences 1987

Longueur inflorescences 1988

Nombre gousses / inflo 1987

Nombre gousses / inflo 1988

Poids de 1000 gousses

Densité février 1989

Carmejane semis 1986

Densité 1 mois après levée

Hauteur 1 mois après levée

Hauteur au 27/04/88

Croissance au 27/03/87

Croissance au 15/04/87

Hauteur à la floraison 1987

Hauteur à la floraison 1988

Hauteur à la floraison 1989

Hauteur $2^{e}$ coupe (25/08/86)

Hauteur $2^{e}$ coupe (29/07/87)

Hauteur $2^{e}$ coupe (13/07/88)

Hauteur $3^{3}$ coupe (29/09/86)

Hauteur $3^{e}$ coupe (04/09/87)

Hauteur $3^{e}$ coupe (22/08/88)

Hauteur $4^{e}$ coupe $(12 / 10 / 87)$

Date de floraison 1987

Date de floraison 1988

Date de floraison 1989

Remontaison au 25/08/86

Remontaison au 29/07/87

Remontaison au 04/09/87

Remontaison au 13/07/88

Remontaison au 22/08/88

Nombre d'inflorescences par mètre 1987

Densité juillet 1989

Variance expliquée par chaque axe

$\begin{array}{rr}-0,572 & -0,309 \\ -0,065 & -0,458 \\ -0,627 & -0,221 \\ -0,878 & 0,302 \\ -0,743 & -0,142 \\ -0,830 & 0,315 \\ -0,804 & -0,400 \\ -0,261 & -0,860 \\ -0,471 & -0,769 \\ -0,766 & -0,398 \\ -0,710 & -0,457 \\ -0,838 & 0,132 \\ -0,930 & 0,114 \\ 0,114 & 0,368 \\ 0,463 & 0,024 \\ 0,390 & 0,462 \\ -0,029 & 0,620 \\ -0,312 & 0,598 \\ -0,667 & 0,451 \\ -0,139 & 0,019 \\ -0,848 & -0,230\end{array}$

$-0,451$

$-0,697$

$-0,797$

$-0,228$

$-0,546$

$-0,794$

$-0,598$

$-0,873$

$-0,950$

$-0,906$

$-0,948$

$-0,514$

$-0,952$

$-0,884$

$-0,673$

$-0,625$

$-0,667$

$-0,470$

$-0,910$

$-0,814$

$-0,898$

$-0,938$

$-0,958$

0,646

$-0,667$

48,8
$-0,368$

$-0,096$

$-0,277$

$-0,753$

$-0,554$

0,524

0,383

0,167

0,160

0,318

0,235

$-0,655$

0,140

$-0,097$

$-0,390$

0,722

0,635

0,664

0,157

0,003

$-0,002$

0,199

0,001

0,210

$-0,285$

16,7

0,287
0,238
0,316
0,205
0,256
0,190
$-0,274$
$-0,092$
$-0,167$
$-0,319$
$-0,432$
$-0,294$
$-0,170$
0,067
0,356
0,371
0,384
0,010
0,080
0,046
$-0,170$

$-0,514$

$-0,293$

$-0,020$

0,028

0,231

0,154

0,077

$-0,109$

$-0,007$

0,136

$-0,024$

$-0,064$

$-0,056$

$-0,007$

0,254

0,371

0,126

$-0,456$

$-0,320$

0,650

$-0,180$

$\begin{array}{rr}0,394 & -0,524 \\ 0,266 & -0,244 \\ -0,215 & 0,204 \\ 0,227 & -0,190 \\ 0,228 & -0,052 \\ 0,111 & 0,048 \\ -0,006 & 0,012 \\ 0,004 & 0,177 \\ 0,179 & 0,080 \\ -0,026 & 0,078 \\ 0,034 & 0,025 \\ 0,297 & 0,212 \\ 0,111 & 0,163 \\ 0,175 & 0,213 \\ 0,355 & 0,284 \\ 0,118 & -0,131 \\ -0,193 & -0,191 \\ -0,274 & -0,250 \\ 0,055 & 0,054 \\ -0,173 & -0,022 \\ -0,045 & 0,141 \\ -0,188 & -0,050 \\ -0,010 & 0,099 \\ 0,429 & -0,225 \\ 0,376 & -0,086\end{array}$

En gras, les coefficients supérieur à 0,7 ; en italique, ceux inférieur à 0,5 . 


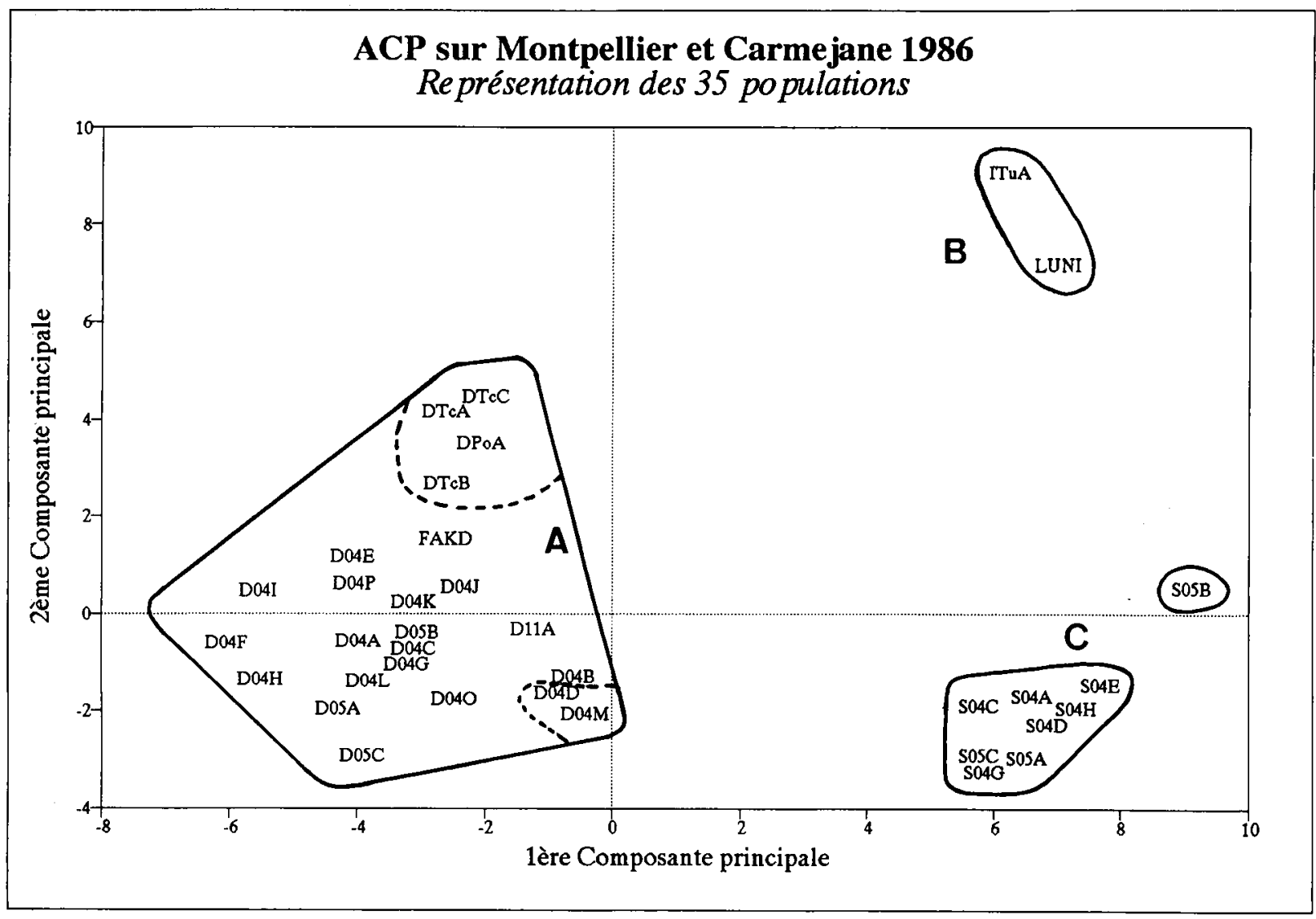

Fig 1. Premier plan de l'analyse en composantes principales réalisée sur 46 variables agronomiques (21 pour Montpellier et 25 pour Carmejane) et enveloppes des classes identifiées. Les origines des variétés de pays sont données sur le tableau I.

La classification hiérarchique réalisée sur la base des distances euclidiennes obtenues à partir des coordonnées issues de l'ACP permet de regrouper les variétés en classes de comportement comparable. Son utilisation commence aussi à se généraliser (Charmet et al, 1989 ; Volaire et al, 1992). Ici, c'est une classification hiérarchique ascendante qui a été réalisée à partir des 4 premières composantes. L'arbre de distances obtenu est présenté sur la figure 2. On note une nette séparation entre les types doubles (groupe $A$ ), les intermédiaires $(B)$ et les simples (C). Pour une plus grande information, nous avons choisi. de diviser l'arbre en 6 classes. Dans ce cas, le rapport de la variance interclasse sur la variance totale est de 0,89 . La classe $A 1$ regroupe les variétés prospectées dans le sudest, à l'exception de 2 d'entre elles (classe A3) et le témoin Fakir. La classe $\mathrm{A} 2$ réunit les origines introduites d'Europe de l'Est (Tchécoslovaquie et Pologne). On note l'isolement de la variété de pays S05B au sein de la classe C1. Ceci est probablement attribuable à la mauvaise qualité des semences (faible levée) et sa faible vigueur générale. La classe $\mathrm{C} 2$ regroupe l'ensemble des autres types simples. Ces classes s'isolent bien sur le plan 1/2 de l'ACP, comme le montre la figure 1. Les moyennes des différentes classes pour les variables sont données sur le tableau VII. En plus des différences générales liées au type de croissance entre les groupes A, B et C, il existe des comportements particuliers des classes. Des différences significatives ont été observées entre ces classes. Ainsi, la classe A3 se caractérise par une excellente croissance hivernale à Montpellier et, à l'inverse, par une plus faible vigueur et pérennité surtout sur le site de Carmejane. Les cultivars introduits d'Europe de l'est (classe A2) sont, en moyenne, les plus tardifs et les moins pérennes, ont une croissance hivernale faible, mais leurs composantes du rendement grainier sont bonnes (nombre de gousses par inflorescence et densité des inflorescences). Comme nous l'avons déjà signalé, les variétés de type simple (classe C2) se distinguent par leur faible vigueur et pérennité. Elles ont plus de tiges florifères, mais chaque inflorescence porte moins de gousses que celle des types doubles. Cette classification servira de base à la constituion de pools géniques en vue 


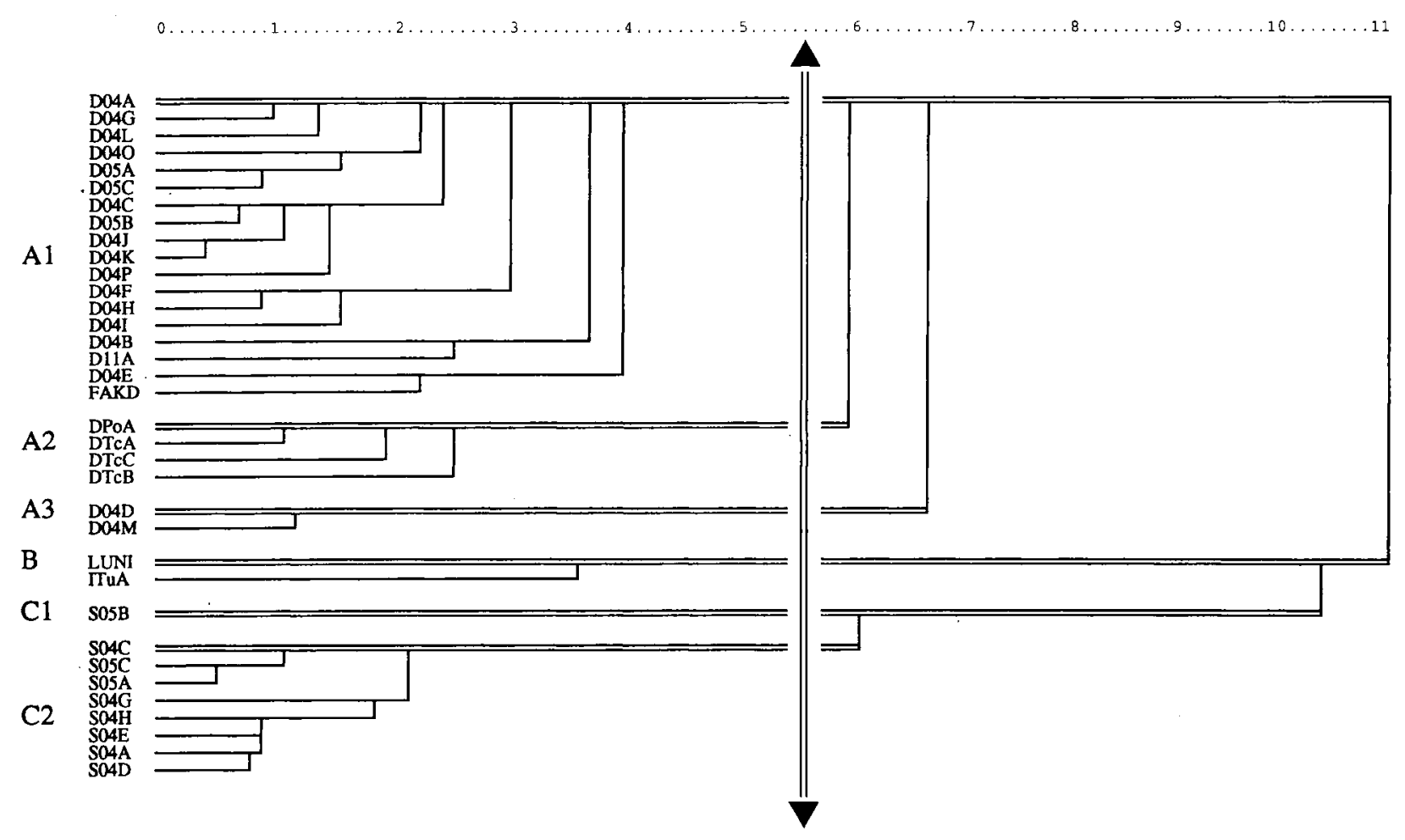

Fig 2. Arbre de distance, basé sur une classification ascendante hiérarchique sur la distance euclidienne mesurée à partir des coordonnées des variétés de pays sur les 4 premiers axes de l'analyse en composantes principales.

de conserver ces ressources génétiques et de valoriser ce matériel par une sortie variétale.

\section{CONCLUSION}

Cette étude a permis de rassembler et d'évaluer des variétés de pays (ressources génétiques) de sainfoin multipliées et conservées par des agriculteurs du sud-est de la France, en dehors de tout programme spécifique de conservation. Ces départements français du sud-est ont une tradition importante de multiplication et de commercialisation de variétés de pays de différentes espèces fourragères (luzerne, sainfoin,...). Ces variétés de pays restent originales par rapport au matériel amélioré et sélectionné disponible sur le marché (cultivars français Fakir ou Lunique, ou cultivars d'Europe de l'est ou de Turquie commercialisés via l'Italie). L'originalité de ces matériels montre bien que les agriculteurs multiplicateurs de ces régions ont su trier, sélectionner et conserver leur propre matériel, comme en témoigne le faible nombre d'erreurs (2) faites lors de l'enquête sur le rattachement des lots de semences fournis au type de croissance.

Les lots collectés avaient été produits sur l'exploitation depuis plusieurs années. Ils étaient en général sales avec des pourcentages importants de graines de mauvaises herbes, notamment de pimprenelle, Sanguisorba minor L, ou de déchets de battage, ces pourcentages ayant pour certains lots dépassé $50 \%$. Plusieurs lots ont eu des facultés germinatives très faibles, ce qui témoigne bien des difficultés de conservation de semences de cette espèce, surtout en conditions non contrôlées à la ferme. Cela explique aussi les faibles densités de levée observées sur certaines origines telles que S05B.

La croissance hivernale de ces variétés de pays est significativement supérieure au témoin de type intermédiaire Lunique et souvent supérieure au témoin de type double Fakir, bien que cette différence soit moins nette et souvent non significative. La différence entre les variétés de pays et les témoins est en revanche plus nette en ce qui concerne le démarrage de printemps. La plupart des variétés de pays démarrent et fleurissent plus tôt que le témoin Fakir. C'est là l'originalité de ce matériel et son intérêt pour les systèmes fourragers méditerranéens, intérêt d'ailleurs fortement souligné par les éleveurs. Cet avantage est d'autant plus marqué que le sainfoin peut être pâturé très tôt au printemps car il ne météorise pas. On note ici une forte complémentarité avec les cultivars de luzerne cultivés 
Tableau VII. Moyenne des différentes classes issues de I'ACP pour les notations réalisées sur les essais de Montpellier et de Carmejane semés en 1986.

\begin{tabular}{|c|c|c|c|c|c|c|}
\hline Classes issues de l'ACP & $A 1$ & $A 2$ & A3 & $B$ & $C 1$ & $C 2$ \\
\hline \multicolumn{7}{|l|}{ Montpellier semis 1986} \\
\hline Densité de levée & 7,3 a & $8,1 \mathrm{a}$ & 7,5 a & $4,2 \mathrm{~b}$ & $2,5 \mathrm{~b}$ & $6,7^{b}$ \\
\hline Volume $1^{\text {re }}$ coupe 86 & 5,3 & 5,3 & 5,3 & 3,6 & 4,5 & 5,6 \\
\hline Volume $2^{\theta}$ coupe 86 & 5,9 a & $5,6 \mathrm{ab}$ & $5,5 a b$ & $4,4 \mathrm{bc}$ & $3,2^{c}$ & $5,1 \mathrm{ab}$ \\
\hline Volume $2^{e}$ coupe 87 & 6,6 a & 7,6 a & $3,8^{b}$ & $3,9 \mathrm{~b}$ & $2,0^{b}$ & $2,4 \mathrm{~b}$ \\
\hline Volume $3^{e}$ coupe 87 & $7,2 \mathrm{a}$ & 6,6 ab & $5,8 \mathrm{bc}$ & $4,7 \mathrm{c}$ & $4,5^{\mathrm{c}}$ & 5,7 bc \\
\hline Volume $1^{\text {re }}$ coupe 88 & $7,5 \mathrm{a}$ & $8,0^{a}$ & $5,3 b c$ & $6,1 \mathrm{~b}$ & $3,8 \mathrm{c}$ & $4,6^{c}$ \\
\hline Volume $1^{\text {re }}$ coupe 89 & 7,2 a & $4,9 \mathrm{~b}$ & 7,8 a & $2,6 \mathrm{c}$ & $2,8 \mathrm{c}$ & $4,1 \mathrm{bc}$ \\
\hline $\mathrm{CH}$ décembre 86 & $7,7 \mathrm{~b}$ & $6,1^{c}$ & $9,0^{a}$ & $3,3 \mathrm{~d}$ & $5,2 \mathrm{c}$ & $7,8 \mathrm{ab}$ \\
\hline $\mathrm{CH}$ février 87 & $7,5 \mathrm{ab}$ & $5,5 \mathrm{c}$ & 8,5 a & 3,4 d & $5,5^{c}$ & $6,7 \mathrm{bc}$ \\
\hline $\mathrm{CH}$ décembre 88 & $7,4^{a}$ & $4,4 \mathrm{~b}$ & $8,0 \mathrm{a}$ & $2,7 \mathrm{~b}$ & $3,8 \mathrm{~b}$ & $3,9 \mathrm{~b}$ \\
\hline $\mathrm{CH}$ février 89 & $6,8^{b}$ & $4,5 \mathrm{c}$ & 9,0 a & $2,3 d$ & $3,0 \mathrm{~cd}$ & $4,0 \mathrm{c}$ \\
\hline Remontaison juillet 87 & $4,9 \mathrm{a}$ & $5,0 \mathrm{a}$ & 5,3 a & $2,1 \mathrm{~b}$ & $1,0 \mathrm{~b}$ & $1,0 \mathrm{~b}$ \\
\hline Remontaïson septembre 87 & $2,0 \mathrm{a}$ & $2,0 \mathrm{a}$ & $2,0 \mathrm{a}$ & $1,3 \mathrm{~b}$ & $1,1 \mathrm{bc}$ & $1,0^{c}$ \\
\hline Nombre Inflo 87 par tige & 3,0 & 3,3 & 3,2 & 3,4 & 3,0 & 3,1 \\
\hline Nombre Inflo 88 par tige & 3,7 & 3,6 & 3,6 & 4,0 & 3,8 & 4,2 \\
\hline Longueur moyenne Inflo 87 & $7,7 \mathrm{~b}$ & $7,7 \mathrm{~b}$ & $6,0 \mathrm{c}$ & 10,3 a & $7,5^{b}$ & $8,2 \mathrm{~b}$ \\
\hline Longueur moyenne Inflo 88 & $7,6 \mathrm{~b}$ & $7,9 \mathrm{~b}$ & $6,0^{\mathrm{c}}$ & 9,5 a & $7,0 \mathrm{bc}$ & 7,2 bc \\
\hline Nombre gousses 87 par Inflo & $11,6 \mathrm{bc}$ & $17,8 \mathrm{a}$ & $13,2 \mathrm{bc}$ & $15,1 \mathrm{ab}$ & $7,0 \mathrm{~d}$ & $9,4 \mathrm{~cd}$ \\
\hline Nombre gousses 88 par Inflo & $15,3 \mathrm{~b}$ & 20,8 a & $14,6 \mathrm{~b}$ & 13,2 bc & $6,4^{d}$ & $9,8 \mathrm{~cd}$ \\
\hline Poids 1000 gousses 87 & 24,6 a & $23,4 \mathrm{ab}$ & $20,7^{b}$ & 24,1 a & $23,7 a b$ & $23,8 \mathrm{ab}$ \\
\hline Densité février 89 & $7,3^{a}$ & $6,6 \mathrm{a}$ & 8,0 a & $3,7 \mathrm{~b}$ & $2,8 \mathrm{~b}$ & $4,5 \mathrm{~b}$ \\
\hline
\end{tabular}

\section{Carmejane semis 1986}

Densité 1 mois après levée

Hauteur 1 mois après levée

Croissance mars 87

Croissance avril 87

Hauteur avril 88

Hauteur floraison 87

Hauteur floraison 88

Hauteur floraison 89

Hauteur $2^{\mathrm{e}}$ coupe 86

Hauteur $2^{\mathrm{e}}$ coupe 87

Hauteur $2^{e}$ coupe 88

Hauteur $3^{e}$ coupe 86

Hauteur $3^{e}$ coupe 87

Hauteur $3^{\circ}$ coupe 88

Hauteur $4^{\mathrm{e}}$ coupe 87

Date de floraison 87

Date de floraison 88

Date de fioraison 89

Remontaison août 86

Remontaison juillet 87

Remontaison septembre 87

Remontaison juillet 88

Remontaison août 88

$\mathrm{Nb}$ Inflo $87 / \mathrm{ml}$

Densité juillet 89

$\begin{array}{cc}53,5^{\mathrm{a}} & 61,7^{\mathrm{a}} \\ 9,6^{\mathrm{ab}} & 11,4^{\mathrm{a}} \\ 5,9^{\mathrm{a}} & 4,6^{\mathrm{a}} \\ 6,4^{\mathrm{a}} & 4,9^{\mathrm{ab}} \\ 37,6^{\mathrm{a}} & 33,8^{\mathrm{ab}} \\ 87,4^{\mathrm{a}} & 92,2^{\mathrm{a}} \\ 92,1^{\mathrm{ab}} & 96,8^{\mathrm{a}} \\ 73,8^{\mathrm{a}} & 67,2^{\mathrm{ab}} \\ 55,1^{\mathrm{a}} & 56,1^{\mathrm{a}} \\ 68,0 \mathrm{a} & 68,2^{\mathrm{a}} \\ 52,8^{\mathrm{a}} & 55,0^{\mathrm{a}} \\ 25,5^{\mathrm{a}} & 21,4^{\mathrm{b}} \\ 53,2^{\mathrm{a}} & 50,6^{\mathrm{a}} \\ 22,1^{\mathrm{a}} & 20,2^{\mathrm{ab}} \\ 24,1^{\mathrm{a}} & 21,3^{\mathrm{ab}} \\ 25,6^{\mathrm{b}} & 31,1^{\mathrm{a}} \\ 22,2^{\mathrm{b}} & 27,0^{\mathrm{a}} \\ 13,5^{\mathrm{b}} & 18,3^{\mathrm{a}} \\ 3,7^{\mathrm{a}} & 3,5^{\mathrm{a}} \\ 2,9^{\mathrm{a}} & 2,7^{\mathrm{a}} \\ 2,0^{\mathrm{a}} & 1,7^{\mathrm{a}} \\ 5,0^{\mathrm{a}} & 5,0^{\mathrm{a}} \\ 3,8^{\mathrm{a}} & 3,2^{\mathrm{a}} \\ 80^{\mathrm{bc}} & 102 \mathrm{a} \\ 3,38^{\mathrm{a}} & 3,15^{\mathrm{a}}\end{array}$

$19,4^{\mathrm{b}}$
$5,0^{\mathrm{c}}$
$1,4^{\mathrm{b}}$
$2,4^{\mathrm{c}}$
$29,5^{\mathrm{b}}$
$89,2^{\mathrm{a}}$
$93,1^{\mathrm{ab}}$
$65,7^{\mathrm{ab}}$
$30,8^{\mathrm{b}}$
$57,8^{\mathrm{b}}$
$36,1^{\mathrm{b}}$
$14,8^{\mathrm{d}}$
$31,3^{\mathrm{bc}}$
$13,0^{\mathrm{c}}$
$13,7^{\mathrm{c}}$
$29,0^{\mathrm{a}}$
$26,7^{\mathrm{a}}$
$19,7^{\mathrm{a}}$
$1,5^{\mathrm{b}}$
$1,3^{\mathrm{b}}$
$1,0^{\mathrm{b}}$
$2,8^{\mathrm{b}}$
$1,0^{\mathrm{a}}$
$102^{\mathrm{a}}$
$1,00^{\mathrm{c}}$

$13,5^{b}$

$52,0^{\text {a }}$

$5,3^{\mathrm{c}} \quad 7,6^{\mathrm{bc}}$

$2,3 \mathrm{~b} \quad 6,2^{\mathrm{a}}$

$3,5 \mathrm{bc} \quad 5,4 \mathrm{ab}$

$31,7^{\mathrm{b}} \quad 31,6^{\mathrm{b}}$

$56,5^{\mathrm{c}} \quad 63,0^{\mathrm{c}}$

$72,3^{\mathrm{c}} \quad 78,0^{\mathrm{bc}}$

$52,7^{\mathrm{c}} \quad 52,9^{\mathrm{c}}$

$19,1^{\mathrm{c}} \quad 26,1^{\mathrm{bc}}$

$31,7^{\mathrm{C}} \quad 35,4^{\mathrm{C}}$

$27,4^{\mathrm{C}} \quad 27,2^{\mathrm{c}}$

$19,6^{c} \quad 23,9 \mathrm{ab}$

$26,8^{\mathrm{c}} \quad 27,7^{\mathrm{c}}$

$16,3 \mathrm{bc} \quad 15,6 \mathrm{bc}$

$20,0^{b} \quad 20,6$ ab

$19,0^{d} \quad 19,2$ d

$14,0^{\mathrm{c}} \quad 12,7^{\mathrm{c}}$

$8,0 \mathrm{c} \quad 7,5 \mathrm{c}$

$1,0^{\mathrm{b}} \quad 1,0 \mathrm{~b}$

$1,0^{b} \quad 1,2^{b}$

$1,0^{\mathrm{b}} \quad 1,0^{\mathrm{b}}$

$1,5^{\mathrm{c}} \quad 1,1^{\mathrm{c}}$

1,0 a $\quad 1,0$ a

89 ab $105^{a}$

$2,00 \mathrm{bc} \quad 2,46 \mathrm{ab}$

La légende des variables est donnée sur les tableaux III et IV. La composition des classes est donnée sur la figure 1. Les valeurs suivies d'une même lettre ne sont pas significativement différentes au seuil de $5 \%$ (test de Duncan). 
dans ces régions et qui démarrent nettement plus tard. Ces différences et cet intérêt ont été constatés par d'autres auteurs dans des régions de climats analogues (Negri et al, 1987).

Le point clef de la sélection du sainfoin et de sa commercialisation reste le faible niveau de multiplication de semences de cette espèce, un maximum de 600 à $800 \mathrm{~kg}$ de graines sont produites pour 50 à $60 \mathrm{~kg}$ de graines semées. Ces origines n'offrent sur ce point aucun avantage puisque la production de semences de l'ensemble des variétés de pays de type double est toujours inférieures à celle de Fakir ou, au mieux, du même niveau. Les niveaux de production de semences des types simples restent, eux, bien inférieurs.

II faut noter le relatif mauvais comportement, dans ces essais, des sainfoins de type simple et notamment leur faible pérennité, à l'inverse de ce qui est observé habituellement. Ceci peut être expliqué, en partie, par le mode de conduite de ces expérimentations. En effet les essais ont été conduits en fauche, avec un rythme lent qui s'adapte parfaitement au cycle de développement des types doubles. Les types simples sont généralement pâturés, dans ces régions, au printemps et à l'automne et bénéficient d'un large repos estival. Ces interactions entre les types de croissance, la pérennité et le pâturage ont déjà été notées par Badoux (1964) et Cooper (1972).

Ce travail d'évaluation et la disponibilité de ces ressources génétiques vont permettre de relancer la sélection de cultivars de sainfoin plus précoces et mieux adaptés aux environnements méditerranéens, sur la base de la constitution de pools issus des classes observées. Néanmoins, it conviendra, pour améliorer le niveau de production de semences, de rechercher d'autres sources de variabilité, si elles existent.

\section{REMERCIEMENTS}

Nous remercions MM $F$ Lechevallier, $M$ Vabre et $E$ Gilly pour leur assistance technique, le lycée agricole de Gap et le parc naturel régional du Queyras pour avoir accepté d'implanter des essais de comportement et de les avoir suivis, la région Provence-AlpesCôte-d'Azur pour avoir financé une partie de ce travail.

\section{RÉFÉRENCES}

Badoux S (1964) Études des caractères morphologiques, physiologiques et agronomiques de populations d'Esparcette (Onobrychis sp). La recherche agronomique en Suisse Vol IV, Fasc 2, 111-190 (1965)

Broucqsault LM, Feraud JP (1990) Le sainfoin dans le sud-est : une carte maîtresse en région difficile. Bulletin FNAMS - Semences 113, 23-24

Carleton AE, Wiesner LE (1968) Production of Sainfoin Seed. In : Sainfoin Symposium at Montana State University, 71-73

Charmet G, Balfourier F (1991) Further evaluation of exotic germplasm of perennial ryegrass for use in French plant breeding programmes. Euphytica 57, 67-76

Charmet G, Bion A, Balfourier F (1989) Agronomic evaluation of perennial ryegrass wild populations from Ireland for use in French plant breeding programmes. agronomie 9, 985-991

Cooper CS (1972) Establishment, hay yield and persistence of two sainfoin growth types seeded alone and with low-growing grasses and legumes. Agron J 64, 379-381

GNIS (1991) La Légumineuse des zones calcaires sèches. Le sainfoin. Groupement national interprofessionnel des semences et plants, Paris

Lapeyronie A (1982) Les productions fourragères méditerranéennes (JJ Maisonneuve, Larose, eds), Paris

Marie R (1961) Point de vue sur le sainfoin. Journée sur le sainfoin. Commission agronomie générale et production fourragère. INRA et CETA du sudAveryon. Document INRA

Negri V, Veronesi F, Falcinelli M (1987) Germplasm evaluation of Onobrychis viciffolia Scop for agronomic traits. Genetic Agraria 41, 25-40

Volaire F, Lelièvre F, Prosperi JM (1992) Production of cultivars and native populations of Trifolium subterraneum $L$ in South of France (Corsica). Aust $J$ Exp Agric 32, 619-625 